\title{
Analysis of passive interference on radio station from AC UHV power
}

\author{
GAN Zheyuan ${ }^{1}$, ZHANG Jiangong ${ }^{1}$, ZHAO Jun ${ }^{1}$ and $L u$ ying $^{2}$ \\ ${ }^{1}$ China Electric Power Research Institute, Wuhan,China \\ ${ }^{2}$ North China Electric Power University, Beijing 102206, China
}

\begin{abstract}
The problems of Electromagnetic Interference in Airborne Intelligence Radar Stations near the AC UHV Transmission Lines is researched in this paper, which is mainly passive interference. And then analyzed the passive interference of AC UHV lines. It mainly studied the influence of the tower and line on the distance detection performance of the air-to-air intelligence radar of the UHV transmission lines. and integrated the influence of the surface reflection surface, the shadowing effect and the secondary radiation echo interference, Proposed a passive interference protection distance for UHV transmission lines and airborne intelligence radar stations
\end{abstract}

\section{1 introduction}

With the rapid development of current science and technology, UHF radio stations(such as analog TV and digital TV broadcasting station, military aviation radars, etc. ), which have strict requirements on the surrounding electromagnetic environment. The solution of passive interference of these stations with UHV transmission lines with large size metal structures has become a research hot spot[1,2].

The air-to-air intelligence radar is the radar which used to search, monitor, identify airborne targets and determine their coordinates and motion parameters, it also known as the air-to-air search radar[3].It is an important electronic technology for acquiring air target information in modern warfare equipment.

In this paper, it analyzed the passive interference of the AC UHV transmission lines, and mainly studied the influence of the tower and line on the distance detection performance of the air-to-air intelligence radar of the UHV transmission lines, and then integrated the influence of the surface reflection surface, the shadowing effect and the secondary radiation echo interference, Proposed a passive interference protection distance for UHV transmission lines and airborne intelligence radar stations.

\section{Analysis of passive interference of transmission lines and airborne radar stations}

The passive interference of transmission lines to radar is mainly manifested in three aspect[4]:
- The secondary radiation of radar electromagnetic waves by obstacles such as transmission lines and towers can generate fixed interference echoes to the radar;

- When the ground facilities such as transmission lines or substations are at a certain distance from the radar, the effective reflection surface of the radar position may be destroyed in this direction, which affects the performance of the radar detection;

- The orientation of the ground facility of the transmission line or substation may cause a loss of shielding from the electromagnetic waves emitted by the radar.

For the meter-band radar, the electric field strength radiated from the antenna to a point in space is the vector sum of the direct wave and the ground reflected wave, and its detection performance is related to the radar position. According to the requirements of Standard $2^{[5]}$, for the meter wave radar, the detection power at different elevation angles can be described by the following formula:

$$
r=R_{0} \cdot F(\alpha) \cdot \sqrt{1+D^{2}|R|^{2}+2 D|R| \cos \left(\frac{4 \pi h}{\lambda} \sin \alpha+\phi\right)}
$$

Where: $R_{0} \longrightarrow$ radar free space nominal distance; $F(\alpha)$ _the vertical direction coefficient of the radar antenna; $D 、 R 、 \phi$ _espectively represent the ground diffusion coefficient, the ground reflection coefficient and the reflected wave lag phase; $h$ _the height of radar antenna; $\lambda$ radar operating wavelength.

When the ground is an ideal reflection condition, the sum of all items under the root number is close to 4 at a certain elevation angle, and the radar detection distance 
can be increased by nearly 1 times after the square root is at a certain elevation angle. It can be seen that the terrain around the radar antenna has a great influence on the radar detection performance. The size of this area is usually calculated according to the Fresnel zone required by the radar elevation angle of the first lobe.

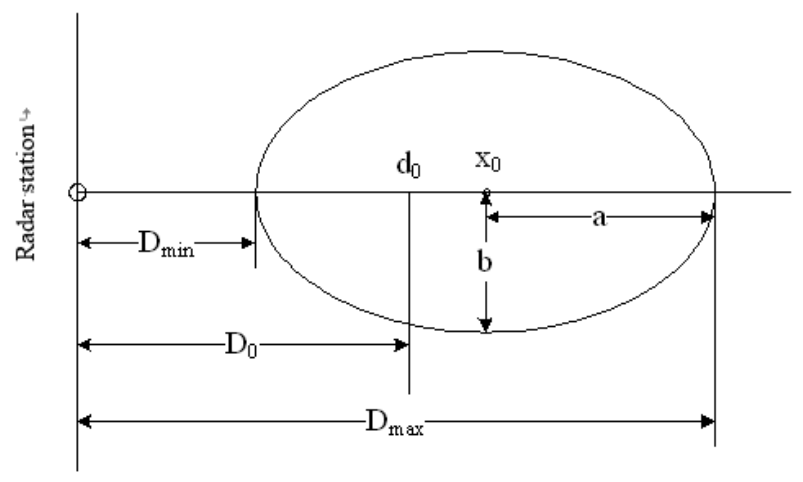

Fig. 1 The first wave of disc center elevation of Fresnel zone

This area is an ellipse, as shown in Figure 1. The relationship of its long axle $a$ 、 short half shaft $b$ 、 Ellipse center ${ }^{x_{0}}$ with radar antenna height $h$ 、 operating wavelength $\lambda$ 、 target elevation angle $\alpha$ is as follows:

$$
\begin{gathered}
x_{0}=\frac{h}{\sin \alpha}\left(1+\frac{\lambda}{2 h \sin \alpha}\right) \\
a=\sqrt{\frac{h \lambda}{\sin ^{3} \alpha}\left(1+\frac{\lambda}{4 h \sin \alpha}\right)}
\end{gathered}
$$

Where: $h$ _the center height of the radar antenna; $\lambda$ _radar operating wavelength; $\alpha$ _the elevation angle on the vertical plane of the antenna.

Then, the farthest reflection distance $D_{\text {max }}$ required by the first lobe elevation angle, the closest reflection distance $D_{\min }$ are:

$$
\begin{aligned}
& D_{\max }=x_{0}+a=\frac{h}{\sin \alpha}\left(1+\frac{\lambda}{2 h \sin \alpha}\right)+ \\
& \frac{2 h}{\sin \alpha} \sqrt{\frac{\lambda}{4 h \sin \alpha}\left(1+\frac{\lambda}{4 h \sin \alpha}\right)} \\
& D_{\min }=x_{0}-a=\frac{h}{\sin \alpha}\left(1+\frac{\lambda}{2 h \sin \alpha}\right)- \\
& \frac{2 h}{\sin \alpha} \sqrt{\frac{\lambda}{4 h \sin \alpha}\left(1+\frac{\lambda}{4 h \sin \alpha}\right)}
\end{aligned}
$$

If the first lobe elevation expression $\alpha_{1}=\arcsin \left(\frac{\lambda}{4 h}\right)$ is brought in (4) and (5), it is simplified and available:

$$
\begin{aligned}
& D_{\text {max }}=23.3 \frac{h^{2}}{\lambda} \\
& D_{\text {min }}=0.7 \frac{h^{2}}{\lambda}
\end{aligned}
$$

The distance between the Geometric reflection center and the radar $d_{0}$ is:

$$
d_{0}=\frac{h}{\tan \alpha}
$$

$$
\begin{gathered}
\text { Put }{ }^{\alpha}{ }_{1} \text { the expression substituted into (8) gives: } \\
d_{0}=4 \frac{h^{2}}{\lambda}
\end{gathered}
$$

As can be seen from the above formula, the distance between each reflection point is proportional to the square of the height of the antenna $h$, and is inversely proportional to the wavelength $\lambda$.According to the analysis: the electromagnetic energy density of the antennas of different distance segments is not the same, and more than $60 \%$ of the energy is concentrated near the center point of geometric reflection. For this reason, when considering the influence of ground obstacles on radar detection performance, it can be calculated according to radar's geometric reflection point. According to stricter requirements, the working frequency of the radar is taken as $300 \mathrm{MHz}$, and the height of the antenna center is taken as $10 \mathrm{~m}$. Substituting the equation (6), (7) and (9) can be obtained: $D_{\max }=2330 \mathrm{~m} 、 D_{\min }=70 \mathrm{~m} 、 d_{0}=400 \mathrm{~m}$, the protection distance of meter wave radar to UHV transmission lines is $2330 \mathrm{~m}$.

Ground facilities such as high-voltage overhead transmission lines may have a shadowing effect on direct waves and ground reflection waves radiated from radar antennas at a certain distance outside the Fresnel zone. Even at a distance other than the farthest point of the effective reflective surface, the ground reflected wave may be shielding, so that the detection performance at a low elevation angle is greatly affected or the vector sum of direct and reflected waves at a certain point in space is affected, as shown in Figure 2. The average height of the UHV transmission line route tower is $50 \mathrm{~m}, d_{0}$ takes $400 \mathrm{~m}$, and the height $h$ of the radar antenna is $10 \mathrm{~m}$ away from the ground. The protection distance between the UHV transmission line and the radar calculated according to Figure 2 is:

$d_{0}+d=d_{0}\left(1+\frac{H}{h}\right)=400\left(1+\frac{50}{10}\right)=2400 m$ 


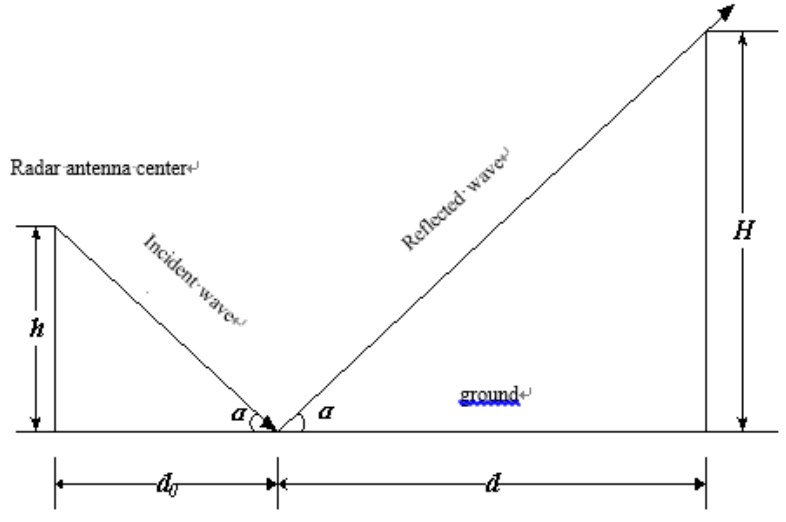

Fig. 2. Schematic diagram of transmission line blocking reflection wave

\section{Simulation test of the influence of transmission lines on radar}

The test of the influence of obstacles such as UHV transmission line routes and towers on radar detection performance is most ideally carried out on actual circuits. Considering that the transmission line and the tower itself are relatively large, it is difficult to carry out the physical test. Therefore, the" scaling ratio" method is used for the simulation test.

According to Wang Qi, Zhao Jie, and Zhao Zhibin's research on the "passive interference of $800 \mathrm{kV}$ DC transmission lines to the radio stations in the short-wave band", the parameters set in this paper are as follows. The experimental prototype is a "drum type" iron tower. The ratio is $30: 1$, the tower height is about $100 \mathrm{~m}$ $(100 / 30 \mathrm{~m}$ in the test), the transmission line adopts 8 split double loop form, the wire splitting pitch is $400 / 30 \mathrm{~mm}$, and the wire type is $8 \times$ LGJ-500/35 aluminum cable steel reinforced. Stranded wire with a wire radius of $16 / 30 \mathrm{~mm}$ and a pitch of $500 / 30 \mathrm{~m}$. The ground wire is aluminum cable steel reinforced with a grounding resistance of no more than $15 \Omega$. During the test, a 3-pitch 4-tower model was selected for testing.

The transmitting antenna and receiving antenna adopt the double-ridged horn antenna with working frequency band of $1 \sim 18 \mathrm{GHz}$, its average gain is $11.3 \mathrm{~dB}$, the impedance is $50 \Omega$, the maximum power is $300 \mathrm{~W}$, and the peak power is $500 \mathrm{~W}$. The power of the transmitted signal source is E8257D with a maximum output power of $10 \mathrm{dBm}$ and a maximum operating frequency of 40GHz. The receiver uses the E8257D spectrum analyzer and its operating frequency range is $9 \mathrm{kHz} \sim 26.5 \mathrm{GHz}$. The signal source and the transmitting antenna and the spectrum analyzer and the receiving antenna are respectively located in the two sides of the transmission line and the tower, the signal source is connected with the transmitting antenna, and the spectrum analyzer is connected with the receiving antenna[3]. The test layout is shown in Figure 3.

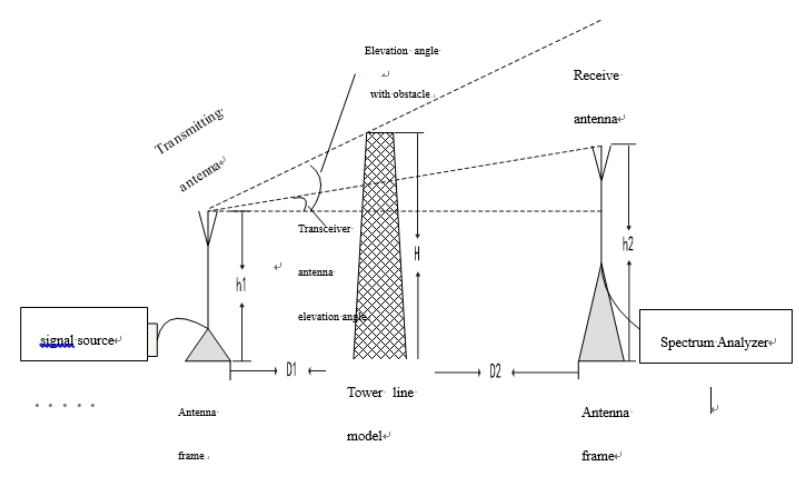

Fig.3 Layout of measurement

Where: $D 1$ _distance between the transmitting antenna and the tower line model, units: $\mathrm{m} ; D 2$ distance between the receiving antenna and the tower line model, units: $\mathrm{m}$; $h 1$ the height of the transmitting line, units: $\mathrm{m} ; H \longrightarrow$ the height of the tower and line model, units: $\mathrm{m} ;{ }^{h 2}$ _the height of the receiving line, units: $\mathrm{m} ; \alpha \longrightarrow$ the elevation angle between the transmitting antenna and the obstruction highest tangent, in degree; $\beta$ - the elevation angle between the connecting line of the transceiver antenna and the horizontal line, in degree.

The main parameters are set as follows: based on the source power and the receiving distance, the distance between the transmitting antenna and the receiving antenna is determined, that is , $(D 1+D 2)$ is $100 \mathrm{~m}$, test frequency selection $3 \mathrm{GHz}, 7 \mathrm{GHz}$ and $12 \mathrm{GHz}$. During the test, follow the steps below.

- Testing the propagation characteristics of electromagnetic waves in free space.

Select the open space, fix the transmitting antenna and receiving antenna at intervals of $100 \mathrm{~m}$, the transmitting antenna frame height is $1.5 \mathrm{~m}$; when the transmitting signal frequency is set to $3 \mathrm{GHz}, 7 \mathrm{GHz}$ and $12 \mathrm{GHz}$ respectively, The corresponding erection height of the receiving antenna is $2.1 \mathrm{~m}, 2.5 \mathrm{~m}, 3 \mathrm{~m}$ and $6 \mathrm{~m}$ respectively. Record the power indicator displayed by the spectrum analyzer;

- Testing the effect of iron tower on the propagation characteristics of electromagnetic waves.

Keep the setting parameters of the spectrum analyzer and the output power of the signal source unchanged, and set the position of the receiving antenna and transmitting antenna unchanged. The tower model is set at $70 \mathrm{~m}, 50 \mathrm{~m}$, $30 \mathrm{~m}$ and $10 \mathrm{~m}$ from the transmitting antenna respectively. The corresponding erection height of the receiving antenna is $2.1 \mathrm{~m}, 2.5 \mathrm{~m}, 3 \mathrm{~m}$ and $6 \mathrm{~m}$ respectively. Read the power indicator displayed by the spectrum analyzer;

- Testing the influence of iron towers and transmission lines in the propagation characteristics of electromagnetic waves.

Keep the setting parameters of the spectrum analyzer and the output power of the signal source unchanged, and set the position of the receiving antenna and the transmitting antenna unchanged. Set the assembled iron tower and transmission line models at positions $70 \mathrm{~m}$, 
$50 \mathrm{~m}, 30 \mathrm{~m}$, and $10 \mathrm{~m}$ away from the transmitting antenna. Record the power indicator displayed by the analyzer.

From the test results, the mainly influence of transmission line is the line, and the tower has less influence on the signal transmission. The results of the passive interference simulation test of the transmission line to the radar station during the 1988 GB1361892"Electromagnetic Environment Protection Requirements for Airborne Radar Stations" are given. The test principle and arrangement are the same as this test. The test results also show that the influence of highvoltage overhead transmission lines on signal propagation is mainly the influence of split conductors, and the influence of towers can be ignored. The main reason is that the actual size of the tower forms a shielding angle to the transmitting antenna smaller than the critical unshielded opening angle.

\section{Conclusion}

In summary, for the meter band radar, the electric field intensity radiated from the antenna to a point in space is the vector sum of the direct wave and the ground reflected wave, its detection performance is related to the radar position, and the path of the ground reflected wave should not be blocked by the ground object. Direct waves and ground reflected waves radiated from the radar antenna may also have a shadowing effect at a certain distance outside the Fresnel zone.

When using a $1000 \mathrm{kV}$ UHV single-circuit transmission line, the guard spacing is $2400 \mathrm{~m}$. If the height of the radar position is significantly higher than the average height of the transmission line conductor, the protection distance of the meter wave radar to satisfy the effective reflection surface is $2400 \mathrm{~m}$, and the remaining radars can satisfy the active interference protection distance of $1600 \mathrm{~m}$. However, before determining the protection distance between the $1000 \mathrm{kV}$ UHV transmission line and the air-to-air radar station, it is also necessary to negotiate with the radar station management department. In the specific implementation, if it is difficult to ensure the protection spacing, other areas can be negotiated and resolved in the case that the main responsibility area is satisfied to meet the above requirements.

\section{Acknowledgement}

This work was supported by the Project of SGCC (GY71-16-011).

\section{References}

1. Yin Hui, Zhang Xiaoming, Wang Yantao, et al. Research on 3D Visualization of Electromagnetic Interference for Ultra-high Voltage Transmission Lines[J]. High Voltage Engineering, 40, 12, 2014.

2. Yang Jiawei, Tang Bo, Huang Li, et al. Research on Interference between UHV Power Transmission Lines and Air Defense Surveillance Radar Station[J]. Journal of China Three Gorges University, 2018.

3. Ding Lufei, Geng Fulu, Chen Jianchun, et a. Radar principles[M]. Beijing. Publishing House of Electronics Industry, 2014.

4. GB13618-92. Electromagnetic Environment Protection Requirements for Airborne Radar Stations[S]. Beijing. Standard Press of China, 1992

5. GJBZ20195-3. Selection Criteria for Military Ground Radar Positions[S]. Beijing. Standard Press of China, 1993 